patients. The most frequent type of ophthalmological affectation was panuveitis with retinal vasculitis in $40 \%$, followed by uveitis in $36 \%$. The median VA at the first visit (RE 0.85 LE 0.85), and at the last follow-up visit (RE 1 LE 1), observing an improvement in VA after follow-up. The most common extra ocular involvement was the presence of oral aphthae in $90 \%$, followed by erythema nodosum and arthritis in $45 \%$. Positivity for HLA B51/B57 was observed in $81 \%$ of the patients. The most frequent immunosuppressant used was CsA $59 \%$, followed by ADA $40 \%$. Among the main aftermath, the presence of cataract was observed in $40 \%$ and synechia in $27 \%$. The mean follow-up of these patients was 85 months. Conclusion: We observed an improvement in VA during the follow-up of these patients compared to VA at the beginning; $70 \%$ of patients continued with immunosuppressive treatment with good control of the disease at more than 85 months of follow-up.

Disclosure of Interests: Jesús Alejandro Valero Jaimes: None declared, Olga Maiz-Alonso Speakers bureau: Pfizer, Ana Carmen Blanco Esteban: None declared, Andrea De Diego Sola: None declared

DOI: 10.1136/annrheumdis-2019-eular.5966

\section{AB0626 \\ INCIDENCE OF SERIOUS INFECTIONS AND PATTERNS OF COTRIMOXAZOLE PROPHYLAXIS IN PATIENTSWITH ANCA-ASSOCIATED VASCULITIDES}

Konstantinos Thomas ${ }^{1}$, Aglaia Chalkia ${ }^{2}$, Dimitrios Drekolias ${ }^{1}$, Christina Tsalapaki ${ }^{1}$, Argyro Lazarini ${ }^{1}$, Kalliopi Klavdianou ${ }^{1}$, Katerina Antonatou ${ }^{1}$, Anastasia Makris ${ }^{1}$, Chrisoula Hatzara ${ }^{1}$, Emilia Hadziyannis ${ }^{1}$, Pinelopi Kouki ${ }^{2}$, Dimitrios Petras ${ }^{2}$ ${ }^{1}$ Dimitrios Vassilopoulos ${ }^{*}{ }^{1}$ Joint Rheumatology Program, National and Kapodistrian University of Athens, School of Medicine, Clinical ImmunologyRheumatology Unit, 2nd Department of Medicine, Athens, Greece; ${ }^{2}$ Nephrology Department, Hippokratio General Hospital, Athens, Greece

Background: Infections are among the most serious complications in patients with ANCA-associated vasculitides (AAV) and contribute significantly in overall mortality.

Objectives: To describe the incidence and risk factors for serious infections, as well as the patterns of cotrimoxazole prophylaxis in AAV patients (GPA and MPA).

Methods: Retrospective, descriptive study of AAV patients followed in a tertiary referral center (Clinical Immunology-Rheumatology Unit and Nephrology department). Patient and disease characteristics treatments and serious infections were recorded.

Results: 56 AAV patients were included (women: 50\%, mean age at diagnosis: 59.8 years, mean disease duration: 5.1 years, GPA: $68 \%$, generalized disease: $82 \%)$. Most frequent organ involvement was renal $(71 \%)$, lung $(68 \%)$, nervous $(20 \%)$, skin $(18 \%)$ and mucous membranes/ eyes (14\%). 21 serious infections were recorded in 16 patients (incidence: 7.2 per 100 patient-years), with respiratory tract infections (43\%) and herpes zoster (19\%) being the most frequent. Incidence was 6.2times higher in patients with severe combined lung-kidney involvement compared to those without (20 vs 3.2 per 100 patient-years, $\mathrm{p}<0.001$ ). Plasma exchange and/or hemodialysis ( $44 \%$ vs $12 \%, p=0.01)$, age $>60$ years $(62 \%$ vs $35 \%)$ and treatment with cyclophosphamide (CYC)/rituximab (RTX) combination ( $38 \%$ vs $3 \%, p=0.001)$ were more common among those who developed a serious infection. Lung-kidney involvement and combined CYC/RTX treatment remained statistically significant in multivariate logistic regression analysis. Cotrimoxazole prophylaxis was given in $70 \%$ of patients treated with CYC and/or RTX $(n=46)$. Patients with combined lung-kidney involvement were more likely to receive prophylaxis $(82 \%$ vs $58 \%, p=0.08)$. No case of pneumocystis pneumonia was diagnosed during follow-up.

Conclusion: In this real-life cohort, more than one out of four AAV patients experienced a serious infection during follow-up, with pneumonia and herpes zoster being the most frequent. Patients with combined lung and kidney involvement had a $\sim 6$ times higher risk for developing a serious infections and were more likely to receive appropriate cotrimoxazole prophylaxis.

Disclosure of Interests: None declared

DOI: 10.1136/annrheumdis-2019-eular.7243

\section{AB0627 \\ INTESTINAL BEHCET'S DISEASE: THE CLINICOPATHOLOGY ANALYSIS OF 38 CASES}

${ }^{1}$ Guosheng Wang ${ }^{*}$, LI Xiaomei', LI Xiangpei ${ }^{1}$, LI Chuanying ${ }^{2} .{ }^{1}$ The First Affiliated Hospital,University of Science and Technology of China; Rheumatology and Immunology, Hefei, China; ${ }^{2}$ The First Affiliated Hospital,University of Science and Technology of China, Pathology, Hefei, China

Background: Due to the rarity of $\mathrm{Gl}$ involvement in $\mathrm{BD}$, the diagnosis of intestinal BD relies on a combination of both vasculitis and IBD type characteristics. Histopathology play an important role in differentiation.

Objectives: To invastigate the clinic features, findings of endoscopy and histopathology of intestinal Behcet's disease.

Methods: The clinical features, findings of endoscopy and histolopathology of 38 intestinal Behcet's disease were analyzed retrospectively.

Results: The mean age of 38 cases were $44.1 \pm 15.6$ years, the mean course were $64.8 \pm 90.4$ months. Typical clinic manifestations and endoscopic finding existed in $24(63.2 \%)$ and $22(57.9 \%)$ patients with intestinal Behcet's disease respectively. The most common(33, 86.8\%) symptoms were abdominal pain and diarrhea. The sites of intestinal ulcer were seen more often(22,57.9\%) at the ileum, ileocecal area, on the ileocecal valve. Most colonoscopic appearance of ulcers were usually well demarcated, oval or round $(28,73.6 \%)$. The suggestive histopathological changes were massive neutrophilic infiltration $(20,52.6 \%)$, inflammatory granuloma $(14,36.8 \%)$ and vasculitis $(8,21 \%)$ especially phlebitis.

Conclusion: The diagnosis of intestinal Behcet's disease depends on the combination of clinic features, endoscopic appearance and histopathological findings. massive neutrophilic infiltration, inflammatory granuloma and vasculitis point to the possibility of intestinal Behcet's disease.

\section{REFERENCES}

[1] Dong Y, Shi G. The synopsis of the 8th international meeting on BD [J]. Chin Med J, 1999, 38(2):135-136

[2] Davatchi F, Assaad Khalil S, Calamia KT, et al. The International Criteria for Behçet's Disease (ICBD) : a collaborative study of 27 countries on the sensitivity and specificity of the new criteria [J]. J Eur Acad Dermatol Venereol, 2014, 28 (3):338-347.

[3] Cheon JH, Kim ES, Shin SJ, et al. Development and validation of novel diagnostic criteria for intestinal Behçet's disease in Korean patients with ileocolonic ulcers [J]. Am J Gastroenterol, 2009, 104 (10):2492-2499.

[4] Valenti S, Gallizzi R, De Vivo D, et al. Intestinal Behçet and Crohn's disease: two sides of the same coin [J]. Pediatric Rheumatology, 2017, 15 (1):33-40.

[5] Sakane T, Takeno M, Suzuki N, Inaba G. Behcet's disease. N Engl J Med [J]. 1999, 341(17):1284-91.

[6] Zhuoli Zhang - Fang He - Yanjun Shi. Behcet's disease seen in China: analysis of 334 cases [J]. Rheumatol Int, 2013, 33 (3):645-648

[7] Park J, Cheon JH, Park YE, et al. Risk factors and outcomes of acute lower gastrointestinal bleeding in intestinal Behcet's disease [J]. Int J Colorectal Dis 2017, 32(5):745-51.

[8] Cheon $\mathrm{JH}, \mathrm{Kim} \mathrm{WH}$. An update on the diagnosis, treatment, and prognosis of intestinal Behc, et's disease [J]. Curr Opin Rheumatol, 2015, 27(1):2431.

[9] Lee SK, Kim BK, Kim TI, et al. Differential diagnosis of intestinal Behcet's disease and Crohn's disease by colonoscopic findings [J]. Endoscopy, 2009, 41(1):9-16

[10] Hayasaki N, Ito M, Suzuki T, et al. Neutrophilic phlebitis is characteristic of intestinal Behcet's disease and simple ulcer syndrome [J]. Histopathology, 2004, 45(4): 377-83.

[11] Demirkesen C, Oz B, Go"ksal S. Behcet's disease: pathology. In: Yazici Y, Yazici H, editors. Behcet's syndrome. New York: Springer; 2010. p. 21543.

Disclosure of Interests: None declared

DOI: 10.1136/annrheumdis-2019-eular.7347

\section{$\mathrm{AB} 0628$ \\ ANALYSIS OF CLINICAL FEATURES OF 15 CASES OF IGG4-RELATED DISEASES}

Ge Yang, Yazhen Su, Gailian Zhang, Liyun Zhang, Ke Xu. Shanxi Dayi Hospital, Tai Yuan, China

Background: IgG4-related disease is an autoimmune disease of unknown cause. 
Objectives: Summarizing the clinical characteristics of IgG4-related diseases to improve the understanding of clinicians and achieve early diagnosis and reasonable treatment.

Methods: The clinical data of 15 patients with IgG4-related diseases diagnosed in our hospital from 2014 to 2018 were retrospectively analyzed. Results: The ratio of male to female was $2: 1$ in 15 patients. The average age of onset was 56.8 years (34 to 84 years of age). From the first symptom to the diagnosis of 8 days to 4.75 years, the most common first symptom in 15 patients was Submandibular gland swelling, pain, anorexia, cough, the most common affected organs are the pancreas, lungs, submandibular glands. There were 11 cases of histopathological examination by surgery or needle biopsy. The histopathology showed interstitial fibrous tissue hyperplasia with more lymphocyte and plasma cell infiltration. Immunohistochemistry showed that the number of IgG4positive cells was $>50 / \mathrm{HPF}$, and IgG4/lgG was $>40 \%$. Two of the 15 patients underwent surgical resection of the affected organ occupying lesions before definitive diagnosis. One patient was treated with mycophenolate mofetil and one patient was given a medium dose of hormone combined with tamoxifen. The remaining 13 patients were treated with simple hormones or combination immunosuppressive agents, including hydroxychloroquine, mycophenolate mofetil, azathioprine, and cyclophosphamide.

Conclusion: The organs involved in IgG4-related diseases are diverse, the clinical manifestations are not specific, and the hormone combined immunosuppressive therapy is effective.

\section{REFERENCES}

[1] Umehara H, Okazaki K, Masaki Y, et al. Comprehensive diagnostic criteria for IgG4-related disease (IgG4-RD), 2011[J]. Japanese Journal of Rheumatology, 2012, 22(1):21-30.

[2] Okazaki K, Kawa S, Kamisawa T, et al. Japanese clinical guidelines for autoimmune pancreatitis[J]. Pancreas, 2009, 38(8):849-866.

Disclosure of Interests: None declared

DOI: 10.1136/annrheumdis-2019-eular.7493

\section{AB0629 CIRCULATING ENDOTHELIAL CELLS MAY BE A MARKER FOR VASCULAR INVOLVEMENT IN BEHCET DISEASE}

Nazife Sule Yasar Bilge ${ }^{1}$, Eren Gunduz ${ }^{1}$, Meltem Akay $^{2}$, Timuçin Kaşifoğlu ${ }^{1}$. ${ }^{1}$ Eskişehir Osmangazi University, Eskişehir, Turkey, ${ }^{2}$ Koç Üniversitesi, Istanbul, Turkey

Background: Circulating endothelial cells (CEC) are defined in conditions which vascular damage is seen in course of disease such as systemic vasculitis, coronary artery disease and chronic renal failure. CEC is thought to be an indicator of vascular damage (1).

Behcet disease (BD) is a systemic vasculitis mostly known with recurrent oral and genital ulceration, uveitis and mucocutaneus lesions. On the other hand vascular involvement such as deep venous thrombosis, cerebral sinus thrombosis and pulmonary artery aneurysm, is an important clinical finding of disease which may cause mortality (2).

Objectives: Our aim in this study was to analyse CEC levels in patients with Behcet disease, to compare them between patients with vascular (Group 1) and mucocutaneous (Group 2) involvement. Also we have compared the results of Behcet patients with patients with thrombosis due to other causes (Group 3) and healthy controls (Group 4). Each group involved 20 participant.

Methods: Blood samples of the patients and healthy controls are drawn into tubes containing etylene-diamine-tetra-acetic acid (EDTA). A panel of monoclonal antibodies, including anti-CD45 to exclude hematopoietic cells, anti-CD31, -CD34, -CD36, -CD105, -CD106, -CD133, and -CD 146 and appropriate analysis gates were used to enumerate resting and activated CECs and circulating and endothelial progenitor cells (CEP).

A hundred microlitre complete blood was added and incubated for 20 minutes at room temperature in the dark. After incubation for 10 minutes with erythrocyte lysing solution at room temperature, centrifugation at $1,800 \mathrm{rpm}$ for 5 minutes was performed. Supernatant was removed and washed with phosphate buffer saline (PBS) for two times. Pellet was resuspended with PBS and 300.000-400.000 cells were counted with FACSCalibur flow cytometry device.

Results: Mean age, sex distribution and duration of disease were similar in all groups (Table 1).
Table 1. Demographic features of the study population

\begin{tabular}{|c|c|c|c|c|c|}
\hline & \multicolumn{4}{|c|}{ Group } & \multirow[t]{3}{*}{$\mathrm{p}$} \\
\hline & Group 1 & Group 2 & Group 3 & Group 4 & \\
\hline & \multicolumn{4}{|c|}{ mean \pm standart deviation } & \\
\hline Age & $43.55 \pm 8.31$ & $46.85 \pm 9.43$ & $42.50 \pm 15.22$ & $41.65 \pm 6.54$ & $0.323^{\star \star}$ \\
\hline Duration of disease & $14.90 \pm 8.69$ & $\begin{array}{r}11.30 \pm 8.80 \\
\mathbf{n}\end{array}$ & (\%) & - & $0.109^{*}$ \\
\hline $\begin{array}{l}\text { Female } \\
\text { Male }\end{array}$ & $\begin{array}{c}9(\% 45.0) \\
11(\% 55.0)\end{array}$ & $\begin{array}{c}12(\% 60.0) \\
8(\% 40.0)\end{array}$ & $\begin{array}{c}11(\% 55.0) \\
9(\% 45.0)\end{array}$ & $\begin{array}{c}14(\% 70.0) \\
6(\% 30.0)\end{array}$ & $0.447^{\star * *}$ \\
\hline
\end{tabular}

* Mann Whitney U Test

** Kruskal Wallis H Test

**** Pearson Chi Square Test

CEC levels did not show a statistically significant difference between al groups. CEPs, activated CECs (aCEC) and resting CECs (rCEC) were also compared between groups. CEPs were higher in Behcet patients with thrombosis similar to patients with thrombosis due to other causes ( $p: 0.042)$. Activated CECs levels did not show a difference between groups ( $p>0.05)$.

Resting CECs are higher in Groups 1 and 3 than Groups 2 and 4. The detailed analysis of CEC, CEP, activated and resting CECs between groups is listed in Table 2.

\begin{tabular}{|c|c|c|c|c|c|c|}
\hline & \multicolumn{4}{|c|}{ Group } & \multirow[t]{3}{*}{ p } & \multirow{3}{*}{$\begin{array}{c}\text { Multi } \\
\text { analysis }\end{array}$} \\
\hline & Group 1 & Group 2 & Group 3 & Group 4 & & \\
\hline & \multicolumn{4}{|c|}{ mean \pm standart deviation } & & \\
\hline CEC & $5.09 \pm 4.7$ & $\begin{array}{c}2.52 \pm \\
2.55\end{array}$ & $4.89 \pm 3.83$ & $4.09 \pm 4.9$ & $0.077^{\star *}$ & - \\
\hline CEP & $\begin{array}{c}13.49 \pm \\
9.38\end{array}$ & $\begin{array}{c}7.62 \pm \\
4.02\end{array}$ & $\begin{array}{c}15.6 \pm \\
11.21\end{array}$ & $\begin{array}{c}13.71 \pm \\
10.1\end{array}$ & $0.042^{\star *}$ & $\begin{array}{l}1-2: 0.015 \\
2-3: 0.009\end{array}$ \\
\hline aCEC & $4.39 \pm 5.78$ & $\begin{array}{c}2.24 \pm \\
2.14\end{array}$ & $\begin{array}{c}8.17 \pm \\
13.26\end{array}$ & $\begin{array}{c}8.78 \pm \\
13.21\end{array}$ & $0.054^{\star *}$ & - \\
\hline rCEC & $6.44 \pm 5.4$ & $\begin{array}{c}5.43 \pm \\
3.49\end{array}$ & $9.03 \pm 7.79$ & $3.52 \pm 2.34$ & $0.007^{\star *}$ & $\begin{array}{c}1-4: 0.034 \\
3-4:<0.001\end{array}$ \\
\hline
\end{tabular}

Conclusion: Increased levels of aCECs may be an indicator of active vascular involvement in BD. But in the current study aCEC levels did not show a difference between groups but none of the patients had active vascular involvement which may cause of this sameness. CEP and resting CEC levels were elevated in both groups of patients with thrombosis. So CEC may be a marker for vascular damage but it is not specific for BD.

\section{REFERENCES}

[1] Woywodt A Circulating endothelial cells in vasculitis and transplantation. Pathophysiol Haemost Thromb. 2003;33:500-2

[2] Kutlay S, Calayoglu R, Boyvat A, Turkcapar N, Sengul S, Keven K, Nergizoglu G. Circulating endothelial cells: a disease activity marker in Behçet's vasculitis. Rheumatol Int 2008;29:159-162

Disclosure of Interests: None declared

DOI: 10.1136/annrheumdis-2019-eular.610

\section{AB0630 1 CLINICAL CHARACTERISTICS ANALYSIS AND LITERATURE REVIEW OF 55 CASES OF PANNICULITIS}

${ }^{1}$ Hui Zheng ${ }^{*}$, LI Rong ${ }^{1}$, Guozhu Che ${ }^{1}$, Liyun Zhang ${ }^{1}$, Huiqin Hao ${ }^{2}$. ${ }^{1}$ Shanxi DaYi Hospital, Rheumatology, Tai Yuan, China; ${ }^{2}$ Shanxi University of Chinese Medicine, Jin Zhong, China

Background: Panniculitis is a heterogeneous inflammatory disease involving subcutaneous fat. It can be divided into different subtypes according to the clinical characteristics and pathological changes of the disease Because the cause of panniculitis is unclear, clinical manifestations are diverse, lack of specificity, early diagnosis is difficult, and misdiagnosis and missed diagnosis are prone to occur.

Objectives: To improve the clinical understanding of the disease by retro spective analysis of 55 cases of patients with panniculitis.

Methods: The hospitalized patients with panniculitis were collected from December2011 to October 2018 in the Shanxi Dayi Hospital Affiliated to Shanxi Medical University. The demographics, clinical manifestations, auxiliary examinations and treatments were analyzed and summarized.

Results: The proportion of males and females in the 55 patients was $1: 2.23$, with an average of 53.3 years (18-82 years). A total of 52 cases 